\title{
Automatic Detection of Laryngeal Pathologies in Records of Sustained Vowels by Means of Mel-Frequency Cepstral Coefficient Parameters and Differentiation of Patients by Sex
}

\author{
R. Fraile ${ }^{a} \quad$ N. Sáenz-Lechón ${ }^{a} \quad$ J.I. Godino-Llorente ${ }^{a} \quad$ V. Osma-Ruiz ${ }^{a}$ \\ C. Fredouille ${ }^{b}$ \\ ${ }^{a}$ Circuits and Systems Engineering Department, EUIT Telecomunicación, Universidad Politécnica de Madrid, \\ Madrid, Spain; ' Laboratoire Informatique d'Avignon, Université d'Avignon, Avignon, France
}

\section{Key Words}

Automatic detection of laryngeal pathologies $\cdot$ Sustained vowels $\cdot$ Mel-frequency cepstral coefficients

\begin{abstract}
Mel-frequency cepstral coefficients (MFCC) have traditionally been used in speaker identification applications. Their use has been extended to speech quality assessment for clinical applications during the last few years. While the significance of such parameters for such an application may not seem clear at first thought, previous research has demonstrated their robustness and statistical significance and, at the same time, their close relationship with glottal noise measurements. This paper includes a review of this parameterization scheme and it analyzes its performance for voice analysis when patients are differentiated by sex. While it is of common use for establishing normative values for traditional voice descriptors (e.g. pitch, jitter, formants), differentiation by sex had not been tested yet for cepstral analysis of voice with clinical purposes. This paper shows that the automatic detection of laryngeal pathology on voice records based on MFCC can significantly improve its performance by means of this prior differentiation by sex.
\end{abstract}

Copyright $\odot 2009$ S. Karger AG, Basel
(C) 2009 S. Karger AG, Basel

1021-7762/09/0613-0146\$26.00/0

Fax +4161306 1234

E-Mail karger@karger.ch

www.karger.com
Accessible online at: www.karger.com/fpl

\section{Introduction}

The social and economic evolution of developed countries during the last few years has led to an increased number of professionals whose working activity greatly depends on the use of their voice. It has been reported that this number has reached one third of the total labor force and, in parallel, that approximately $30 \%$ of the population suffer from some kind of voice disorder during their lives [1]. In this context, the relative relevance of voice function assessment among health care activities should increase in the near future and the development of computer-aided software for voice assessment [2-4] should not only support such growth, but also help assessment tasks to get out of the clinics and closer to people's residences and working environments. Additionally, the objective nature of computer-based assessment algorithms has the potential advantage over human-based perceptual evaluation of requiring much less evaluation time and cost in human resources [5].

The computational capacities of computers nowadays allow the combination of information coming from dif-

This research was carried out within a project funded by the Ministry of Science and Technology of Spain (TEC2006-12887-C02) as well as within the framework of the European COST Action 2103. 
ferent sources for processing. In the case of voice assessment, such sources may be as diverse as images from laryngoscopes, acoustic data from voice recordings, or electrical information from electroglottography. However, the use of audio recordings on their own, though it implies dismissing relevant information that may be present in other sources of data, allows noninvasive and easy data collection [6]. As a consequence, this requires less specialization in the use of medical devices and, therefore, eases the task of voice assessment for family doctors and in nonclinical environments.

In this context, current software for automatic voice assessment [2-4] analyzes audio recordings, either previously stored or captured through a microphone, to compute a set of acoustic parameters for which normative values exist and have been published [6,7]. Commonly, such audio recordings correspond to the phonation of sustained vowels, since the whole paradigm relies on the assumption that a healthy person is able to produce sustained sound that is stationary from the signal processing point of view, while the presence of some kind of dysphonia implies the appearance of nonstationarities (e.g. pitch changes, noise related to air turbulences). Bearing this in mind, the calculated acoustic parameters act as perturbation measures and their use for clinical purposes was proposed more than one and a half decades ago [2]. Later on, it was shown that the use of these parameters together with pattern classification techniques as the basis for performing automatic voice assessment could lead to success rates in the range of $90 \%$ in the detection of the presence of laryngeal pathologies [8].

In medical practice, the values of acoustic parameters extracted from voice have been found to be highly correlated with the presence of some laryngeal pathologies [9], thus confirming the previously mentioned assumption. In contrast, some studies also indicate that these objective acoustic measures do not correlate very well with perceptual voice quality measures [10]. This fact, on the one hand, shows the limitations of the analyzed parameters but, on the other hand, it also indicates that both are complementary approaches.

During the last few years, research efforts in speech processing for clinical applications have been evolving the objective voice assessment based on acoustic measures towards:

- improving the classification success rates of automatic pathology detection by means of combining different kinds of parameters [11] and incorporating pattern classification techniques used in other applications of speech analysis [12];

Automatic Detection of Laryngeal Pathologies by MFCC
- introducing alternative parameterization schemes that either capture different features of voice [13] or allow for processing of running speech [14], thus achieving better performance;

- introducing alternative parameters that are more robust against noise and other distortions [15] and avoid the need of pitch detection, which is a difficult task in many circumstances [16]. This difficulty has specifically been observed in the analysis of dysphonic voices [17].

This paper reports on a research work carried out in the third direction. Specifically, it refers to the use of analysis in the cepstral domain for automatic detection of laryngeal pathology. The application of mel-frequency cepstral coefficients (MFCC) to the automatic assessment of voice was first proposed by Dibazar et al. [18] and Godino-Llorente and Gómez-Vilda [19]. The use of MFCC for voice analysis stems from the field of speaker identification and it was first supported more by empirical evidence than by theoretical reasoning. Two additional practical reasons supported this choice: calculation of MFCC does not require pitch detection and these parameters have been shown to be fairly robust against some kinds of voice distortion [15]. In addition, it has been argued that analysis in the cepstral domain for this application is justified by the presence of noise level information in the cepstrum [20] and that MFCC give a compression of this information on the first part of the cepstrum, hence providing some dimensionality reduction and easing the task of pattern classifiers [21]. Thus, a theoretical basis for the application of MFCC to objective voice assessment exists now.

Within the field of speaker identification, MFCC are used to build voice feature vectors that pass through a classification stage that intends to associate the MFCC vector with a template corresponding to a specific speaker. When building such templates, it is common to differentiate between male and female speakers, since such differentiation has shown to improve identification results [22]. A priori, the use of this differentiation also seems reasonable for pathology detection. As a matter of fact, normative values of classical perturbation parameters differ between men and women $[6,7]$ as they also differ in the case of other objective voice quality measurements [23]. Results reported in Ma and Yiu [17] confirm such divergence in normative values between male and female voices and, in addition, they even show a differentiation in the relevance of each acoustic parameter for dysphonia detection.

From a medical point of view, there are factors that support this distinction, such as age [24] and hormonal chang-

Folia Phoniatr Logop 2009;61:146-152 
es [25]. These affect men and women very differently and they even result in different effects on the 'intrasex' variability of voice among normophonic speakers. The modelling work reported by Horáček et al. [26] demonstrates that the anatomic and physiological differences between men and women also result in a different risk of developing some vocal fold pathologies. It must be noted that while the performance of automatic pathology detection decreases when patients are differentiated by sex [27], the herein proposed scheme involves introducing 'a priori' knowledge about sex in order to improve pathology detection.

\section{Materials and Methods}

For this analysis, the speech record database commercialized by the American company Kay Elemetrics is used [28]; more specifically, a subset containing 53 normal (21 male and 32 female) and 173 pathological (70 male and 103 female) records, each consisting of a sustained phonation (1-3 s long) of the vowel /a/ [29]. This subset covers a wide variety of pathologies and the distribution of speakers in age ranges from 26 to 59 years in the case of men and from 21 to 52 in the case of women. The sampling rate of speech records has been made equal to $25 \mathrm{kHz}$, while coding has a resolution of 16 bits. This subset has been chosen because it has an acceptably balanced distribution in age and sex and it covers a good variety of pathologies. For further reference on the present pathologies, the reader can refer to Parsa and Jamieson [29].

\section{Mel-Frequency Cepstral Coefficients}

There are several ways of formulating and calculating MFCC [30]. For this work, the formulation described by Fraile et al. [21] has been used and it is qualitatively described next.

The first step in processing has been to split each voice record in 20 -ms frames with $50 \%$ overlapping between consecutive frames. This short-term processing is justified since detecting the nonstationary nature on pathological voices, as mentioned before, requires avoiding long-term measurements. For the shortterm processing algorithms, the framework provided by Deller et al. [31] has been considered.

The short-term processing, in this case, has a collateral benefit. Since the utilized voice database has approximately three times more pathological than normal records but each normal record is three times longer than each pathological record, the short-term processing results in a balanced number of normal and pathological voice frames, which is important for training the classification scheme.

After splitting the voice signal in frames, each frame undergoes a discrete Fourier transform (DFT), hence computing a short-term DFT of each voice record [31]. This transforms the signal into frequency domain. The left graph of figure 1 shows (in gray) the modulus of the DFT corresponding to one speech frame. It is well known that this function results from the superposition of two effects: the rapid variations of the graph, coming from the quasi-periodic vibrations of the vocal folds, and the slow variations or spectral envelope, mainly related with the specific form of the glottal wave and the resonances induced by the vocal tract.
The second step of MFCC calculation is spectrum smoothing. This smoothing is carried out using a set of filters that mimic the perceptual behavior of the human auditory system. This choice of filters results in a higher spectral resolution at lower frequency bands, where the most significant information for dysphonia detection is contained [32], and it performs better than a set of regularly spaced filters [21]. The main effect of spectrum smoothing is removing the harmonics of the spectrum while keeping an estimate of the spectral envelope (this is the black dashed line in the same graph). The minimum values of this envelope are related to the aperiodicities or nonstationarities of voice, since for a perfectly periodic signal these values should be zero.

The logarithmic operation that comes afterwards and the inverse DFT that transforms the signal from spectral domain into the cepstral domain allow representing the form of the spectral envelope in the first part of the cepstrum (fig. 1). For this reason, the first coefficients of the cepstrum keep information on glottal noise [20]. Moreover, the specific spectrum smoothing carried out in MFCC computation further concentrates information on the first cepstral coefficients [21]. Statistical confirmation of this can be found in Godino-Llorente et al. [33].

Implementation of the above-described cepstral analysis allows converting each 20 -ms voice frame, which at a sample frequency of $25 \mathrm{kHz}$ is formed by 500 samples, into a feature vector having an optimal length of 15-20 MFCC [21]. For the experiments reported in this paper, the length of the feature vector has been chosen to be 16 .

\section{Classification Scheme}

For the purpose of developing the automatic scheme for pathology detection, each feature vector, corresponding to one frame and consisting of 16 MFCC, has been assigned a pair of likelihoods:

- the likelihood of belonging to the phonation of a healthy person $\left(p_{n}^{i}\right)$, and

- the likelihood of that person having pathology $\left(p_{p}^{i}\right)$,

where $i$ indicates the frame index. For normal frames, $p_{n}^{i}=1$ and $p_{p}^{i}=0$, while for pathological frames, $p_{n}^{i}=0$ and $p_{p}^{i}=1$. Therefore, the objective of the automatic system is to produce estimates $\hat{p}_{n}^{i}$ and $\hat{p}_{p}^{i}$ of the previous likelihoods that are as similar as possible to their real values. In the event of processing an unknown voice, the pair $\left(\hat{p}_{n}^{i}, \hat{p}_{p}^{i}\right)$ should give the clinician an indication of the likelihood that such voice belongs to a person having laryngeal pathology.

For the herein reported work, the automatic system has been chosen to have the structure of a multilayer perceptron (MLP). This is a type of artificial neural network whose architecture is outlined in figure 2 . The artificial neural network is an information-processing system composed of computational nodes (circles in fig. 2). Starting from the values of the input feature vector, it weights them with a set of coefficients $\omega_{i j k}$, combines the weighted values among them and with certain constants $\theta_{i j}$ and transforms the combination with a nonlinear function. The outputs of the first stage of computational nodes may then serve as the feeding of a second stage of nodes and the outputs of the last stage are taken to be the outputs of the whole system. This automatic system, before performing detection tasks, has to undergo a training phase that consists in finding values for every $\omega_{i j k}$ and $\theta_{i j}$ suitable for such a task. For a comprehensive review of the artificial neural network and learning algorithms, the reader may refer to Haykin [34]. 
Fig. 1. Graphical interpretation of the

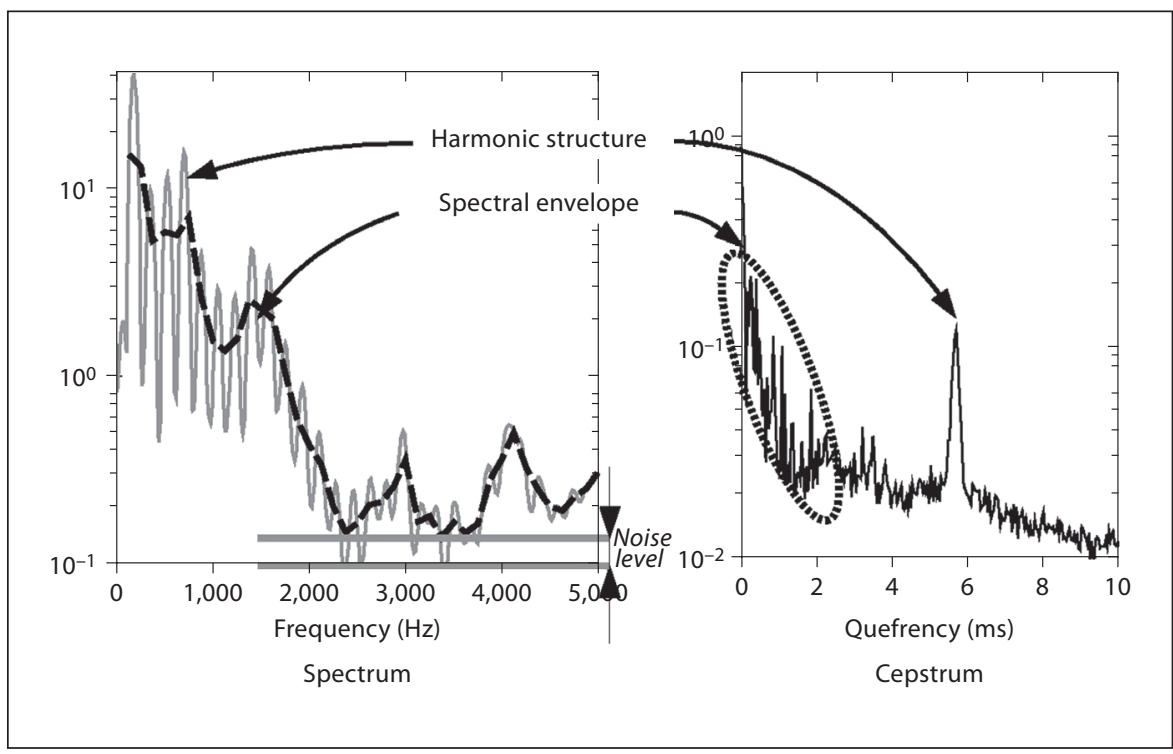
cepstrum.

Fig. 2. Internal structure of an artificial neural network.

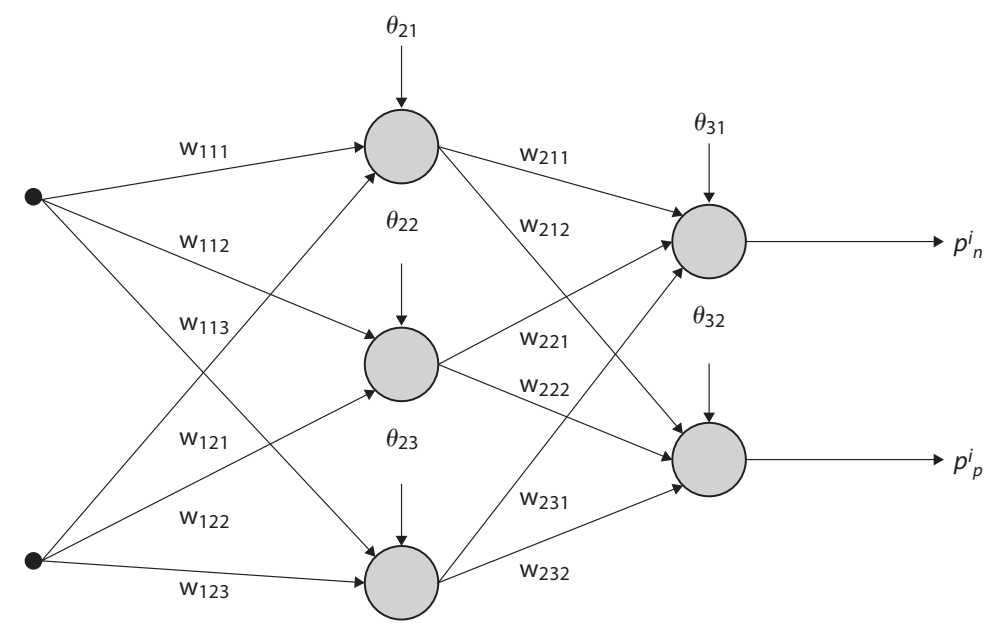

Input values

Hidden nodes

Output nodes

Output values

In this specific case, the chosen architecture for the MLP is as follows: 16 inputs, one corresponding to each MFCC of the feature vector describing the speech frame, 40 hidden nodes and 2 output nodes, corresponding to the 2 outputs $\hat{p}_{n}^{i}$ and $\hat{p}_{p}^{i}$. Using such a system, a set of classification experiments have been run that consist in choosing $60 \%$ of the database records for training the MLP, $10 \%$ for cross-validation during training in order to stop the learning process and the remaining 30\% for testing. Experiments have been repeated with different, randomly chosen, training, validation and testing sets until the resulting classification error rates exhibited confidence intervals below $1 \%$ of their values.

As for classification decisions, if they had to be made at the frame level, they should be based on the estimates $\left(\hat{p}_{n}^{i}, \hat{p}_{p}^{i}\right)$ ob- tained at the output of the MLP classifier. A common way to decide is to base the decision on the log-likelihood ratio $\left(L L R^{i}\right)$ that relates both estimates [35]:

$$
L L R^{i}=\log \frac{p_{n}^{i}}{p_{p}^{i}} \gtrless \theta_{f}
$$

However, for a voice record consisting of $N$ frames, the decision should be made at the record level (in fact, all frames of a record belong to the same voice). The usual way to do this is to use the mean log-likelihood [35]:

$$
L L R=\frac{1}{N} \cdot \sum_{i=1}^{N} \log \frac{p_{n}^{i}}{p_{p}^{i}} \gtrless \theta_{r}
$$


Fig. 3. a Experimental probability density function of the most relevant MFCC for normal and pathological, male and female voices. b Detection error if classification was based only on this parameter, as a function of the decision threshold.

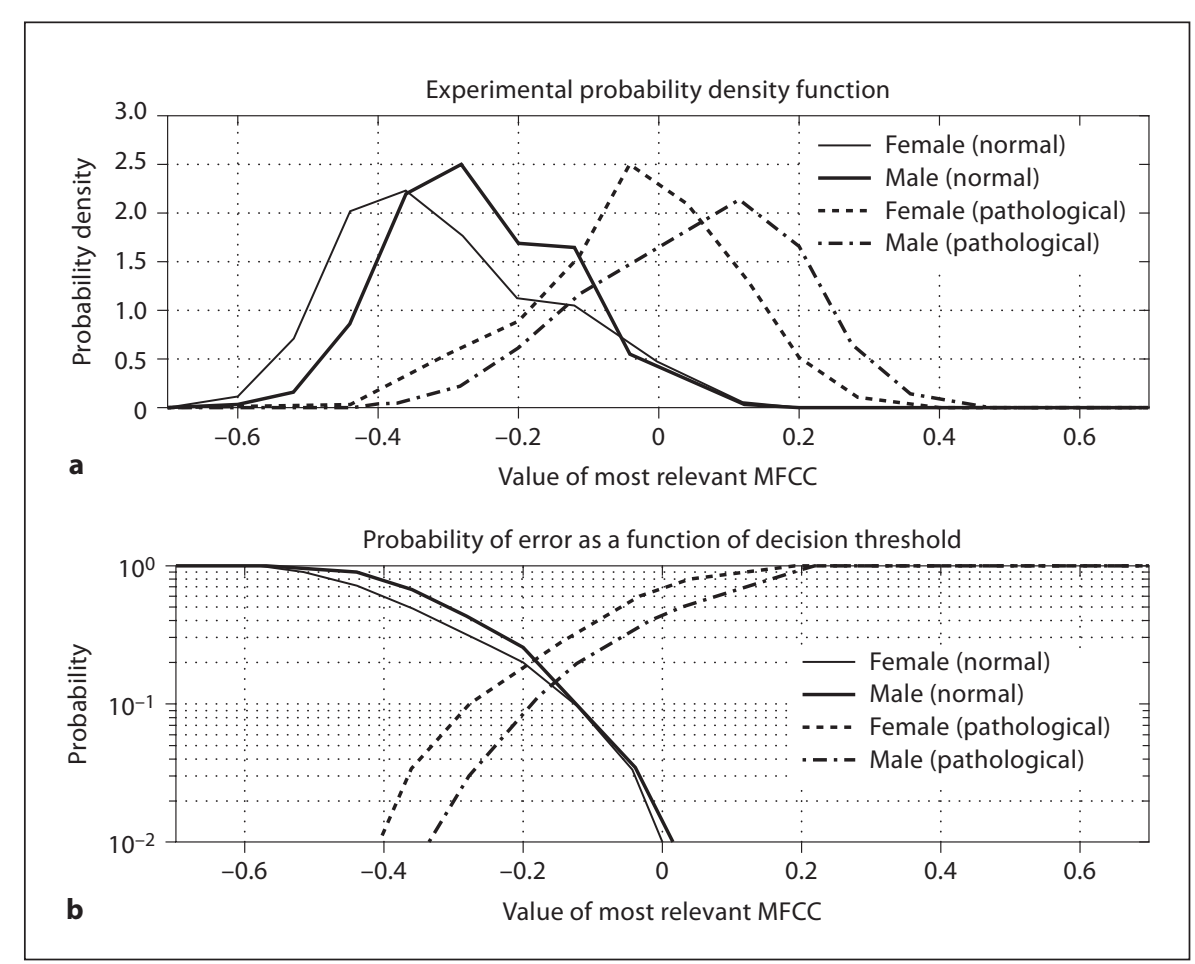

The performance of the detector will obviously depend on both the training process of the system and on the choice of the threshold values $\theta_{f}$ or $\theta_{r}$ that discriminate between normal and pathological voices.

\section{Results}

Within the previously described MFCC feature vectors, each component has a different level of relevance for pathology detection. The most significant components tend to be the first cepstral coefficients [33]. Bearing this in mind, the first analysis that has been carried out consists in studying the sex-specific distribution of the most significant MFCC for normal and pathological speech frames within the available database. Figure 3 shows the resulting experimental probability density functions (fig. 3a). Also, the error rate that would be obtained for each class based on a single-feature classifier, depending on the threshold chosen for decision, is shown in figure $3 \mathrm{~b}$. The most relevant aspects of the graphs are as follows.

- The distribution of the values of the most relevant MFCC for men and women are shifted with respect to each other. Therefore, an independent analysis would lead to different optimal threshold values. This can be easily noticed in figure 3 a.
- The distribution of values for men is narrower than the distribution for women. This results in a lower error rate for male voices at the working point that produces equal error rates for normal and pathological voices, as can be seen in figure $3 \mathrm{~b}$.

Based on these observations, it can be expected that if male and female voices were differentiated before their objective assessment, the results of such an assessment should be more accurate, provided that the procedure was previously tuned differently for each group. As a matter of fact, results obtained with the classification scheme described in the previous section confirm the previous observations. Classification errors obtained for $\theta_{r}=0$ are summarized in table 1 , while the DET plots [36] in figure 4 provide a graphical representation of these results that accounts for variability on the decision thresholds. As expected, male-specific classification produces significantly better results than female-specific classification. In other words, pathology detection based on MFCC is easier for men than for women. This is related to the normal patterns of women's voices having a wider distribution than those of men (fig. 3a). In addition, results also indicate that sex-specific classification can lead to some improvements in the aggregate performance with respect to the case of non-a-priori differentiation by sex (sex-independent classification). 
Fig. 4. DET curves for mixed and separated male and female voices for both frame level and record level classification.

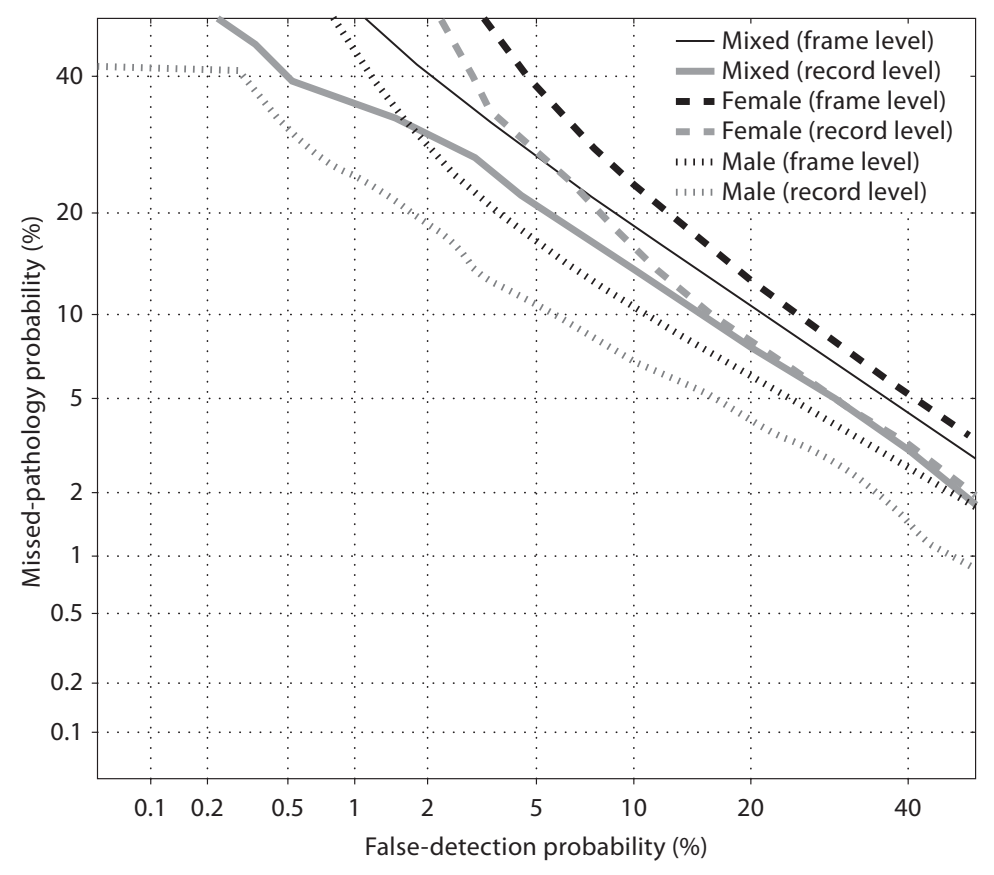

lence noise. Moreover, it achieves that with a reduced set of parameters, hence easing subsequent processing by pattern classifiers.

In addition, the performance of these parameters for automatic pathology detection has been tested and, in contrast with other previously published studies, the impact of considering a priori information on the sex of speakers has been assessed. Results of the reported experiments indicate that sex is a relevant factor when automatically assessing the presence of laryngeal pathologies in speech records corresponding to sustained vowels. More specifically, for cepstral domain analysis it seems that female voices have wider distributions and, thus, they are more difficult to classify. This fact may be due to the greater variability that the voice of women between 20 and 60 years experiences compared to men within the same age range due to aging and hormonal issues [25]. While these results should be confirmed with different data sets, there seems to be a biological justification for them.

On the other hand, the results also indicate that the difference in the distribution of MFCC between men and women is large enough to justify the design of sexspecific classifiers in order to achieve better performance. 


\section{References}

1 Sdersten M, Lindhe C: Voice ergonomics An overview of recent research. Proc 39th Nordic Ergonomics Soc Conf, Lysekil, 2007.

2 Deliyski DD: Acoustic model and evaluation of pathological voice production. Proc 3rd Conf Speech Commun Technol, Berlin, 1993, pp 1969-1972.

3 Boersma P, Weenink D: Praat: Doing Phonetics by Computer. Version 4.3.14. 2005 .

-4 Godino-Llorente JI, Sáenz-Lechón N, OsmaRuiz V, Aguilera-Navarro S, Gómez-Vilda P: An integrated tool for the diagnosis of voice disorders. Med Eng Phys 2006;28:276-289.

5 Bonastre JF, Fredouille C, Ghio A, Giovanni A, Pouchoulin G, Revis J, Teston B, Yu P: Complementary approaches for voice disorder assessment. Interspeech 2007, 8th Annu Conf Int Speech Commun Assoc, Antwerp, 2007, pp 1194-1197.

6 Baken RJ, Orlikoff R: Clinical Measurement of Speech and Voice, ed 2. San Diego, Singular Publishing Group, 2000.

7 Jackson-Menaldi MCA: La voz patológica. Buenos Aires, Editorial Médica Panamericana, 2002.

8 Boyanov B, Hadjitodorov S: Acoustic analysis of pathological voices. A voice analysis system for the screening of laryngeal diseases. IEEE Eng Med Biol Mag 1997;16:74-82.

$\checkmark 9$ Perrin E, Berger-Vachon C, Kauffmann I, Collet L: Acoustical recognition of laryngeal pathology using the fundamental frequency and the first three formants of vowels. Med Biol Eng Comput 1997;35:361-368.

10 Finizia C, Dotevall H, Lundström E, Lindström J: Acoustic and perceptual evaluation of voice and speech quality a study of patients with laryngeal cancer treated with laryngectomy vs irradiation. Arch Otolaryngol Head Neck Surg 1999;125:157-163.

11 Yu P, Ouaknine M, Revis J, Giovanni A: Objective voice analysis for dysphonic patients: a multiparametric protocol including acoustic and aerodynamic measurements. J Voice 2001; 15:529-542.

12 Fredouille C, Pouchoulin G, Bonastre JF, Azzarello M, Giovanni A, Ghio A: Application of automatic speaker recognition techniques to pathological voice assessment (dysphonia) Interspeech 2005, 9th Eur Conf Speech Commun Technol, Lisboa, 2005, pp 149-152.

13 Alonso JB, de Leon J, Alonso I, Ferrer MA: Automatic detection of pathologies in the voice by HOS based parameters. EURASIP J Appl Signal Processing 2001;4:275-284.
14 Umapathy K, Krishnan S, Parsa V, Jamieson DG: Discrimination of pathological voices using a time-frequency approach. IEEE Trans Biomed Eng 2005;52:421-430.

15 Fraile R, Godino-Llorente JI, Sáenz-Lechón N, Osma-Ruiz V, Fredouille C: MFCC-based remote pathology detection on speech transmitted through the telephone channel. Impact of linear distortions: band limitation, frequency response and noise. Proc Biosignals, Porto, 2009.

16 Boyanov B, Ivanov T, Hadjitodorov S, Chollet G: Robust hybrid pitch detector. Electron Lett 1993;29:1924-1926.

17 Ma EPM, Yiu EML: Suitability of acoustic perturbation measures in analysing periodic and nearly periodic voice signals. Folia Phoniatr Logop 2005;57:38-47.

18 Dibazar AA, Narayanan S, Berger TW: Feature analysis for automatic detection of pathological signal. Proc Second Joint EMBS/ BMES Conf, Houston, 2002, pp 182-183.

19 Godino-Llorente JI, Gómez-Vilda P: Automatic detection of voice impairments by means of short-term cepstral parameters and neural network based detectors. IEEE Trans Biomed Eng 2004;51:380-384.

20 Murphy PJ, Akande OO: Quantification of glottal and voiced speech harmonics-tonoise ratios using cepstral-based estimation. Proc 3rd Int Conf Non-Linear Speech Processing (NOLISP'05), Barcelona, 2005, pp 224-232.

21 Fraile R, Sáenz-Lechón N, Godino-Llorente JI, Osma-Ruiz V, Gómez-Vilda P: Use of melfrequency cepstral coefficients for automatic pathology detection on sustained vowel phonations: mathematical and statistical justification. Proc 4th Int Symp Image/Video Commun Fixed and Mobile Networks, Bilbao, 2008.

22 Campbell JB: Speaker recognition: a tutorial. Proc IEEE 1997;85:1437-1462.

23 Hakkesteegt MM, Brocaar MP, Wieringa $\mathrm{MH}$, Feenstra L: Influence of age and gender on the dysphonia severity index. A study of normative values. Folia Phoniatr Logop 2006;58:264-273.

24 Linville SE, Rens J: Vocal tract resonance analysis of aging voice using long-term average spectra. J Voice 2001;15:323-330.

25 Benninger MS, Abitbol J: Voice. Dysphonia and the aging voice; in American Academy of Otolaryngology Head and Neck Surgery Foundation: Geriatric Care Otolaryngology Online. American Academy of Otolaryngology Head and Neck Surgery Foundation, 2006, chapt 3, pp 66-85. http://www.etnet. org/EducationAndResearch/geriatricOtolaryngology.cfm.
26 Horáček J, Laukkanen AM, Šidlof P, Murphy P, Švec JG: Comparison of acceleration and impact stress as possible loading factors in phonation. A computer modeling study. Folia Phoniatr Logop, in press.

27 Umapathy K, Krishnan S: Feature analysis of pathological speech signals using local discriminant bases technique. Med Biol Eng Comput 2005;43:457-464.

28 Massachusetts Eye and Ear Infirmary: Voice Disorders Database. Version 1.03. Lincoln Park, Kay Elemetrics Corporation, 1994.

29 Parsa V, Jamieson DG: Identification of pathological voices using glottal noise measures. J Speech Lang Hear Res 2000;43:469485.

30 Ganchev T, Fakotakis N, Kokkinakis G: Comparative evaluation of various MFCC implementations on the speaker verification task. Proc 10th Int Conf Speech Comput (SPECOM 2005), Patras, 2005, pp 191-194.

31 Deller JR, Proakis JG, Hansen JHL: DiscreteTime Processing of Speech Signals. New York, Macmillan Publishing Company, 1993.

32 Pouchoulin G, Fredouille C, Bonastre JF, Ghio A, Giovanni A: Frequency study for the characterisation of the dysphonic voices. Interspeech 2007, 8th Annu Conf Int Speech Commun Assoc, Antwerp, 2007, pp 1198 1201.

33 Godino-Llorente JI, Gómez-Vilda P, BlancoVelasco M: Dimensionality reduction of a pathological voice quality assessment system based on Gaussian mixture models and short-term cepstral parameters. IEEE Trans Biomed Eng 2006;53:1943-1953.

34 Haykin S: Neural Networks: A Comprehensive Foundation, ed 1. New York, Macmillan College Publishing Company, 1994.

- 35 Bimbot F, Bonastre JF, Fredouille C, Gravier G, Magrin-Chagnolleau I, Meignier S, Merlin T, Ortega-Garcia J, Petrovska D, Reynolds DA: A tutorial on text-independent speaker verification. EURASIP J Appl Signal Processing 2004;2004:430-451.

36 Martin A, Doddington G, Kamm T, Ordowski M, Przybocki M: The DET curve in assessment of detection task performance. Proc 5th Eur Conf Speech Commun Technol (Eurospeech 1997), Rhodes, 1997, pp 18951898. 